\title{
KNOWLEDGE MANAGEMENT: THE TRUTH BEHIND THE CONCEPT
}

\author{
Sidney Viana ${ }^{1}$, Luis Vellacich ${ }^{2}$, Marcos Miranda ${ }^{1}$ and Carolina Pereira ${ }^{1}$ \\ ${ }^{1}$ Itaipu Technological Park, Hernandarias, Paraguay \\ ${ }^{2}$ Itaipu Binacional, Hernandarias, Paraguay
}

\begin{abstract}
Knowledge has become the most precious asset of any organization, being the main ingredient for the success and innovation of the products and services offered. Currently there are different proposals of Knowledge Management models, with the implications of implementing them. Most of them are unrealistic in the context of the organizations and their implementation can be summarized in the document management. Consequently, in a short period of time, they fail in their attempt to adequately manage this precious asset, information. In context, this work proposes an efficient implementation of Knowledge Management, based on a model of four interrelated axes, successfully implemented in a real and complex environment: The Dam Safety project. This project is divided into three phases, where the above-mentioned axes are detailed. It also describes the difficulties encountered throughout the implementation, the actions taken to overcome these difficulties, the results and experiences obtained in the first phase of the project, ending with proposals for improvements for its second phase. The results are very encouraging and have the approval of the final users, who, for many years, were part of the search for a solution that could satisfactorily provide Knowledge Management in their work context.
\end{abstract}

\section{KEYWORDS}

Knowledge Management, Knowledge Management Models, Innovation, Dams

\section{INTRODUCTION}

Knowledge has characteristics of a commodity, which adds value to products or services and becomes one of the basic components necessary for market evolution. The knowledge resulting from the experience acquired over time, also known as tacit knowledge, has an added value that tends to increasing, according to the different experiences lived by people.

However, explicit knowledge is the materialization of knowledge. Generally, its value is despised over time, as experiences are embodied in documents, reports, articles, thesis, books, and other artifacts. It is also known as a static source of information. So, it can generate knowledge projected into the future, becoming current knowledge or obsolete knowledge.

Explicit knowledge is a very important mechanism for transmitting knowledge to others and when it is associated with technology, it becomes efficient. For example, the Internet is a way of capturing information at high speed, capable of providing large volumes of information, which must be classified to obtain those that are reliable and useful to knowledge. Figure 1 shows the data that are the raw material for information. The information, contextualized and with quality, associated with a domain, points to knowledge. The last one generates information that subsequently results in stored data.

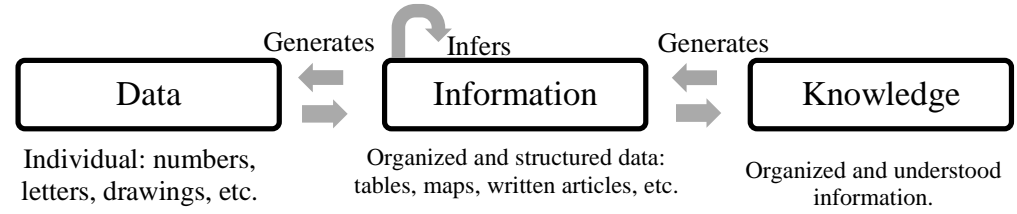

Figure 1. Evolutionary cycle of knowledge 


\section{STATE OF THE ART}

A variety of Knowledge Management models exist in literature. Below are some of the Knowledge Management (KM) models and a brief description of them (Pérez, et. al, 2004; Espinoza, 2006):

The knowledge - creator organization (Nonaka and Takeuchi, 1995): It is based on the mobilization and conversion of tacit knowledge (epistemological dimension) and the creation of organizational knowledge versus individual knowledge (ontological dimension). It is a cyclical and infinite model that contemplates five phases: sharing tacit knowledge, creating concepts, justifying concepts, building an archetype, and expanding knowledge. Basically, they propose the creation of knowledge maps, self-organizing teams, and group dialogue sessions, where individuals reveal and share their tacit knowledge with the rest of the group, through schemes, models, metaphors, and analogies. The people involved in the process of creation and management of knowledge, will be part of the so-called knowledge-creating team, which will consist of knowledge practitioners, knowledge engineers and knowledge officers.

Knowledge Management as a "humanist" vision (Marulanda Echeverry, et. al, 2012): In the words of its author: "[...] it focuses its operation on the commitment of the organization's workforce, in such a way that, where others have emphasized technology as the basis of a system for managing knowledge, here the person, his or her stability within the organization and his or her involvement and alignment with the general objectives and the organizational project, are given prime importance". The model is made up of four phases: management consulting, organizational consulting, implementation of knowledge management plans and verification and monitoring measures. It proposes the development of knowledge maps, establishment of practice communities, creation of a knowledge warehouses, discussion forums, meetings and seminars. The people involved are members of the organization, internal experts, and external experts.

Knowledge Management from the organizational culture (Marsal and Molina, 2002): It is based on the type of organizational culture existing in the institution. It is also composed of five phases based on studying, interpreting, and changing, if necessary, the organizational culture: Self-diagnosis, strategic management, definition and application of the KM model, change management and indicators to measure the impact of KM. They propose the elaboration of yellow pages, learning communities, good practices, assistance and help meetings. Participants: KM managers (people with communication, technological and management skills); members of the organization.

Knowledge Management in education (Sallis and Jones, 2013): They start from the fact that each educational organization should possess and build its own structure, its own KM system, based on its characteristics, its strengths, and weaknesses. KM in education, is a model of KM centered on education centers, fundamentally higher-level education. The phases that make up the model are classification of knowledge, reference framework for KM, knowledge audit, knowledge measurement, technology and knowledge management and knowledge exploitation. They propose knowledge maps, creation and development of virtual communities and collaborative work. The different educational agents participate in the conception, planning and development of the KM system of their own institution.

Gamification in Knowledge Management Systems (Murawski, 2020): In the last decade, gamification has experienced a major popularity boom. Murawski summarized existing human resource management (HRM) related literature of gamification and systemized the findings in this regard into four potential application areas of gamification, being (1) supporting employee motivation, engagement, and performance, (2) improving training outcomes, (3) supporting talent management measures, and (4) foresting knowledge management activities. He found that within these categories, the use of game design elements to enhance motivation, engagement, and performance within the workplace is the most used area of focus.

As Murawski explains, current proponents of gamification emphasize the importance of holistic approaches to gamification design, considering the employee's needs and characteristics, as they have major impact on the effectiveness of the use of game design elements. It must be mention that the use of gamification in the knowledge management system for The Dam Security must take a long time, because it would be necessary develop different kinds of materials to represent the knowledge presented in books, documents, and in the expert's tacit knowledge, considering the existence of fluid communication among the stakeholders. Gamification has proven to work in the specific area of application. As the mentioned author explains, research on gamification in HRM lacks rigor in a few major aspects. He explains that HR scientists are at the beginning of a journey to explore the phenomenon of gamification. Therefore, larges companies, with well-defined policies, a cost-benefit evaluation should be carried out to determine if it is appropriate to implement a knowledge management system applying gamification techniques. 


\subsection{Difficulties and Limitations}

The main constraints and difficulties that can be encountered during the implementation of a KM project are (Pérez et. al, 2004; Jennex, 2005; Rodríguez, 2006; Jennex et. al, 2014 ):

- Lack of objectives: The absence of objectives for KM or the lack of clarity is an impediment to the success of a project. In fact KM, is merely a way to achieve organizational goals, make decisions, or resolve problems and conflicts identified in the organization.

- Lack of planning: this is not an exclusive problem with KM projects, but it is obviously something that must be taken into consideration. The novelty and complexity of KM processes causes organizations to focus and invest a lot of resources in pilot planning and forget the subsequent extension of that project to the rest of the organization.

- Diffuse responsibility: KM initiatives can be headed to failure if a set of responsibilities are not clarified or established among people who are responsible for the entire process. A dedicated KM team is recommended for the design, development, and evaluation of the KM process.

- Contextualization: KM projects cannot be generalized; they must be designed according to the characteristics of the organization (values and objectives, structure, relational system, and organizational functions).

- Conceptual confusion: many organizations tend to confuse information management with knowledge management and use technology (e.g., intranets) potentially valid for KM processes, such as simple organizational document stores.

- Lack of an adequate culture: the absence of an adequate culture for KM leads irremediably to the failure of every effort.

- Absence of the Specialist: Knowledge Management should consider the role of the specialist as an essential element in the orientation of the knowledge base in the domain of the problem or the requirements to be outlined in the organization.

Information management can be defined as the set of activities carried out in order to control, store and, subsequently, adequately recover the information produced, received, or retained by any organization in its operative state. At the heart of information management is the be management of documentation (the information that is reflected in documents) (Bustelo and Amarilla, 2001).

The data source must be reliable; the data must be of quality and verifiable. Choosing good references requires experience and insight. The efficiency of Knowledge Management requires a technological component, a group of technicians dedicated to the task of management, one or more specialists in the domain of knowledge and very well-defined processes that guarantee the maintenance of management over time.

The following section describes the corporate environment considered to carry out Knowledge Management practices. In this environment, initiatives such as Knowledge Management did not have the credibility of high authorities since it had gone through its fourth attempt, made by commercial companies that did not achieve results that justified the financial investments made (Saz, 2001; Ruesta and Iglesias, 2001). Having a solution that meets the challenges and expectations of the requesting company is essential to follow up on the many projects that can be carried out in this area of science.

\section{DESCRIPTION OF THE ENVIRONMENT}

In a long-established organization, thousands of documents are usually produced each year, containing explicit knowledge accumulated over the course of the organization's history.

In the context of dams, small or large hydroelectric plants, this knowledge comes from two important stages: the first one refers to the construction, and the next one refers to the maintenance of the structure. In such stages, tacit knowledge of great importance is generated, mainly in the initial stage, due to the experiences accumulated in the construction phase. Not all constructions steps have been documented, those documentations generated was very simple, lack of techniques support. So, having the support of a technician that participate at the early stage of operations it would be very luck.

Currently, it's very hard to find an enterprise that has the courage to invest an amount of financial resource in a task force to deal with Corporate Knowledge Management. To attempt these demand, most of them take advantage of Document Management System, which allows access to the digital version of the documents 
created in the above-mentioned stages. However, these systems turn out to be document repositories (i.e. database engine), generating the need for specific knowledge (search engine by keywords). These systems are not optimal for dealing with the current problems faced by power generation companies, since the origin of the problems may be at the beginning of the work. For examples, if someone needs information about "crack in the wall", in detail, with explanation's video, photos, similar problems occurred on others Dam, the theory necessary to understand the problem and all technical report on the affected area and others information, could be informed by the System's modules, at the Figure 3.a.

The challenge is to minimize the time needed to transfer the tacit knowledge of senior officials of an organization, to the younger ones. This is because it has taken decades for such officials to accumulate the technical knowledge they now use. In addition, this problem is aggravated once they begin their retirement process, discovering the fact that there are no professionals prepared with the minimum necessary knowledge and do not have the time required to prepare a training program and delivering it, due to their daily activities.

On the other hand, staff members who participate in work scales activities need to constantly remember the hundred similar tasks they performed in the past. However, there is concern about probably mistakes face the work overload occurrence, caused by lack of technical reports information elaborated by the newest technicians, personnel actualization, and contextual technical changes among other reasons. Look for the personal adequate to receive the right information (tacit and explicit knowledge in an efficient and agile way), at the right time is a challenge (Papoutsoglou, et. al, 2017). As a result of lack of cumulative daily knowledge management, in its broad spectrum, this work presents an approach for Knowledge Management modeling and your correspondence implementation. Furthermore, it is generic and therefore applicable to other cases related to other areas of knowledge.

\section{SOLUTION PROPOSAL}

The proposed solution (Figure 3.a) consists of a continuous process, which begins with the capture of knowledge; this is based on technical reports available in information management systems, specialist personal notes and tacit knowledge from experts and technical users who can contribute with recent information.

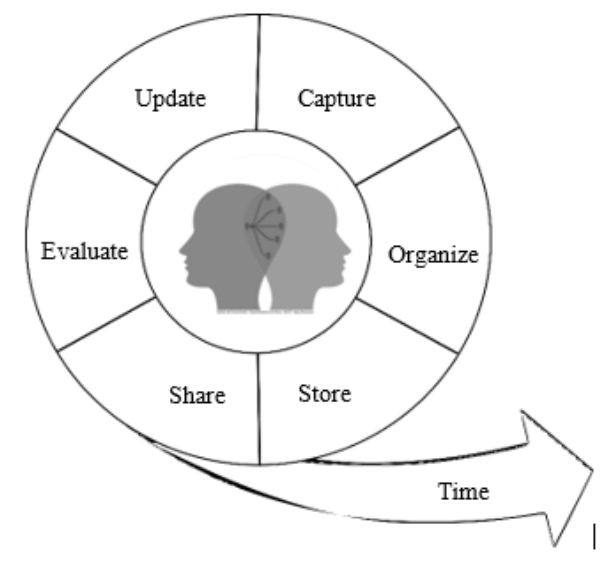

Figure 2. Knowledge management processes adopted

Subsequently, such knowledge is organized into correlative ideas, and classified according to the degree of importance and complexity, by a three-level taxonomy (basic, intermediate, and advanced). Once the information is organized, it is stored through the usage of Information and Communication Technologies (ICTs), and shared, so that it is easily accessible through personal computers and mobile devices. This information is shared with all those involved, so that they can make evaluations, suggestions and test the efficiency of the system in terms of its performance.

The information visualized by the end user is a multimedia document, composed of information in three (3) dimensions, with movement, added audio and a system that facilitates the search for information by subject and complexity. This process allows reductions in time for the explanation process of any content. 
Once a presented topic was understood, it is possible to say that the knowledge was transferred to the end user. Finally, the next step is to update the information, or add new information. This process is carried out continuously so that knowledge lasts through time.

The proposed solution requires support tasks to validate the process. One of them is measurement the knowledge acquired by the end user. In other words, it is necessary to guarantee that the user evolves his knowledge on the subject, through time, and for this, the role of his superior in the organization is fundamental. In the same way, the mentioned user must have the information available in any place of the organization and at any time (through internet connection). In the end, learning related to state of mind.

\subsection{Knowledge Management Model (4 Axes)}

The proposal of a Knowledge Management Model for real problems, is based on four (4) interrelated axes. These axes are (i) the specialist's knowledge, (ii) information and communication technologies, (iii) organizational culture, and (iv) time.

Specialist's Knowledge: The first axis of the model covers the main asset of the organization, which is knowledge. Explicit knowledge is easier to deal with, once it is within everyone's reach. In this sense, the internal order of all this documentation is fundamental. Regarding tacit knowledge, it is provided by the specialist, who is usually the senior employee of the company, and sometimes, retired role. However, those who have not yet done so, are not willing to undertake the task of reviewing documentation and translating it into a language understandable to all. In addition, they do not have the necessary time to take on this new task. The senior available, must have a set of basic characteristics to play the role, such as the ability to share experiences with younger people, good communication skills and good writing skills.

Information and Communication Technologies: Technology is a way and not an end to the knowledge management process. Choosing the right technology is critical, because it must support the changes that will occur over time. One alternative is to use free technologies, which are not necessarily the most appropriate. This is because it can be discontinued, and it will probably be necessary to migrate to another platform. It is important considering the cost/benefit ratio of this. An important factor to be considered is the amount of accesses that must be supported by the system, especially when supporting multimedia data. Therefore, optimal performance is required from the hardware used to maintain the information.

Organizational Culture: The organizational culture is affected by users and their processes. For the end user to have an active participation throughout this transfer of knowledge, it is necessary that he commits himself to it, through his experience, his reviews, and comments in general. When the management process is imposed on the end user, rejection usually occurs when feeling pressure from the imposition. The user must participate through his creative process, at the moment he identifies with the system, understanding that his participation is a way of continuous improvement, tending to participate more often. Some of the processes that can be applied are: (i) Classification of information and knowledge by the specialist. (ii) Validation of the documents generated by the end users. (iii) Inclusion of end users, both in the validation and improvement of the solution.

Time: This must last as long as the knowledge management process is used. Mechanisms must be sought to guarantee actions over time. In the proposed system, time is very well framed through the participation of the superior or boss, where he verifies that knowledge tests were performed, information was updated, periodic meetings with technicians to know what should be added or improved on the proposed environment, among other actions.

The connection between the model and the Systems developed to support de Knowledge Management is about take into account the methodology used to catch the data, organize it and analyze whom will take advantage from it. To offer the information in a strategic and intuitive way, the Systems contain a lot of perspective that complement the user knowledge in any scalable perspective, practical cases, simulations, quiz (supervised by the manager) and searching tool. The manager knows who accessed de Systems, the grade obtained at the quiz (Papoutsoglou, 2017).

\subsection{Computer System Modules}

The basis of the proposed solution is a centralized computer system, where knowledge is available on a web environment and is accessed from both mobile devices and desktop computers. Figure 3.a shows the 
architecture of the solution. The Systems is supported by .NET framework, C\# Language, ASP.NET y MVC architecture. The KM teams include a Dam specialist (retired employee), Solidworks technician, PMI Manager, among others. The specialist deeply knows the needs and the value of the business since worked last decade before him retired. He has accessed the information, translated many sheets into audiovisual and pleasant explanation, changing the reading on paper documents by visual navigation (Figure 3.a and Figure 4). The results obtained is delivered in business values. The feedback helped us to improve for the next delivered. All the time, technicians feed the system with more previously selected information that in turn notifies the end users involved in the topic, in a cyclic way.

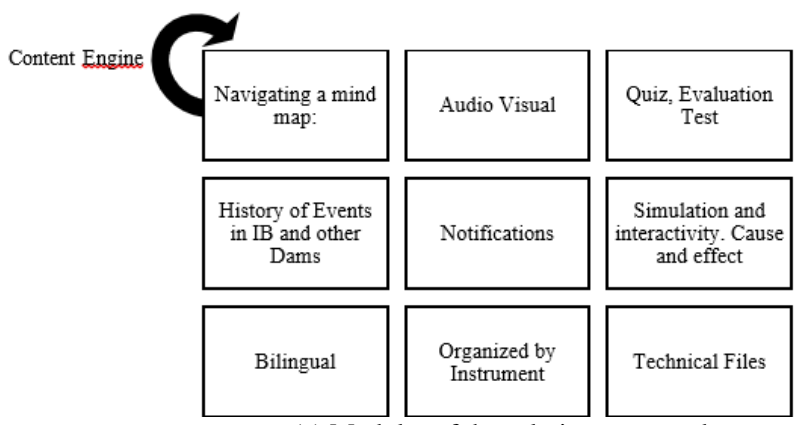

(a) Modules of the solution presented.

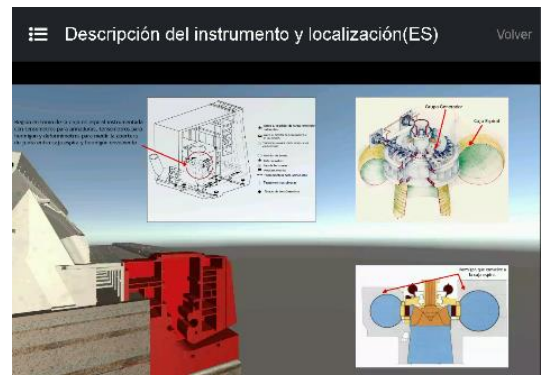

(b) Audio visual material generated.

Figure 3. Presented solution

The computer system is composed of a set of ten (10) modules (Figure 3.a). Each of these modules is briefly described below.

- Web navigation: Structured content according to levels, basic, intermediate, and advanced. Navigation occurs in an intuitive way, facilitating access to different types of content. Each content can be repeated as many times as necessary, until the user feels capable enough to continue advancing with the content.

- Audio visual: The content is presented in an audio-visual way, facilitating the transfer of knowledge (see Figure 3.b). It is understood that the user does not have time to read technical information or understand certain drawings in representation of three (3) dimensions. For this reason, representations with animation and audio strongly help the user's understanding.

- Quiz, evaluation test: Contains an evaluation of the knowledge acquired. In this way it is possible to know if there was knowledge retention. These results are sent to the person in charge who has the objective of guaranteeing the active participation of the majority of the employees.

- Simulation and interactivity: Cause and effect: The simulation and interactivity cause-effect is an environment where the user can play with variables on models of the problem to understand the participation and the importance of each one of them. For example, what is the effect of increasing temperature, or pressure, among other variables.

- Technical files: Access to technical files produced by the organization. These files are technical references in the organization. From the system you can access this information for the purpose of complementing the knowledge in question.

- Organized by instrument: The instruments are rated according to the data they capture from the dam. Some are more important than others. Users can organize their studies according to these priorities.

- Bilingual: Possibility of learning in two languages: Spanish and Portuguese.

- History of events in Itaipu Binational and other Dams: To complement the knowledge of the organization's officials. Examples external and correlated to the area of operation, such as disasters occurred, are very important, so as to prevent this from happening in their future work environment.

- Notifications: System of notifications of the latest content updates and news in general.

- Content engine: Enabling the use of filters to search for specific content as it shown in Figure 4.b. 


\section{DISCUSSION}

The solution presents a knowledge transfer methodology based on the use of multimedia data, in a highly interactive and easy to navigate environment, since it is based on web navigation. It is possible to identify the evolution of knowledge by the user. The base of this solution was the previous information about the working environment of the final users and the knowledge of their daily routine. It was understood that proposing a solution that introduces a degree of difficulty on their activities could lead the user to stop using it. This is because everything is known to have a degree of priority, and the system cannot compete with the priority of their tasks. Another important point was to consider that the end user should use the system in the organization, otherwise there would be no commitment on his part. Figure 4.a shows one of the results of this content creation process, where it can be seen that extensive (top left margin), and sometimes complex (top right margin) documentation is transformed into much more attractive content for the user (animated three-dimensional image with audio).

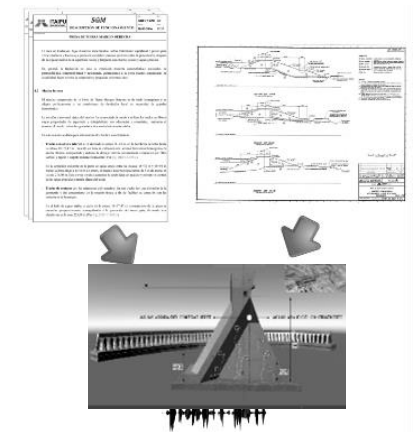

(a) Audio-visual content creation from documentation.

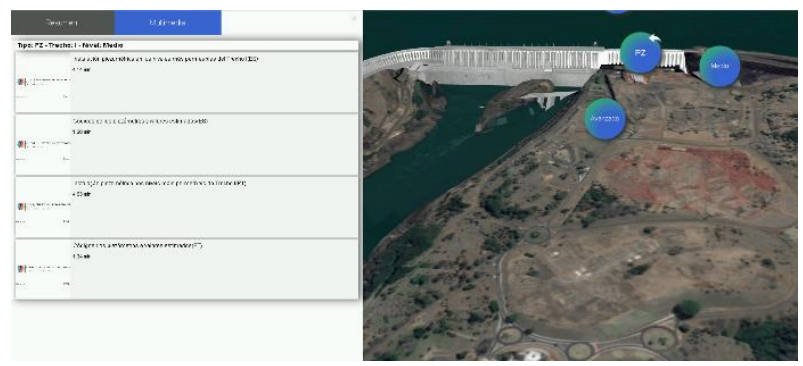

(b) Result by context search engine.

Figure 4. Audio-visual content creation from technical documentation

\section{RESULTS}

The proposed Knowledge Management system was evaluated, considering a Dam staff member as a population. A non-probabilistic or targeted sample was taken based on the representativeness of the population, made up of 14 system users (ranging from 23 to 50 years of age), officials from the Technical Area of the mentioned Dam staff with experience ranging from 1 to 10 years. Grouping was made according to: (P1) focus given to the tool's content, (P2) Distribution of the tool's content, (P3) interaction with the KM Systems tool, and (P4) usefulness of the KM tool in terms of user experience.

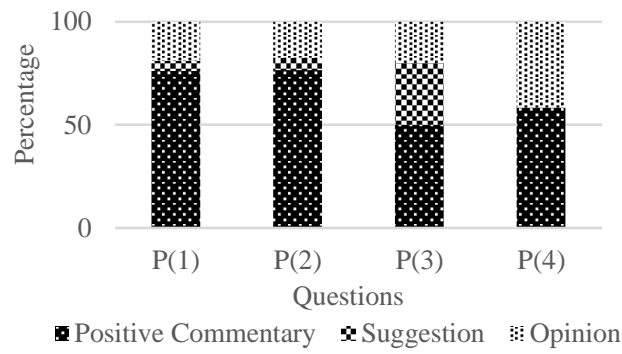

Figure 5. Test results applied to the computer systems users

Tests to the proposed Knowledge Management System were conducted through open questions posed to professionals in the area with extensive experience. 
Considering the groupings described above, four levels of responses were obtained which were classified in the following level: basic, intermediate, advanced, and general. Finally, each one of them has been labeled according to its content as: positive comment, suggestion, and opinion.

The results obtained are summarized in the figure 5. As can be seen, a large percentage of positive comments from the users has been obtained.

\section{CONCLUSION AND FUTURE WORK}

The proposed solution is adaptable to different areas of human knowledge and can be expanded to other branches of knowledge such as medicine, physics, and administration, among others. The proposed solution is based on the presence of the specialist, who knows the domain of the problem and has the skills to carry out the challenge and the technicians who are committed to participate actively throughout this process.

Technology is important but not fundamental, as there are many options available, all depending on the resources required for this priority. The threats lie in the fact that the representatives of the organizations do not take the decision in time to solve a very serious problem that is the loss of senior staff members who do not intend to return to the organization to participate in Knowledge Management programs. This is an intangible but real problem that generates millions of dollars, resulting from the loss of information.

The results achieved so far were very satisfactory and motivating at the same time. One of the most important results was the recovery of tacit information from retired employees that provided vital information for the understanding of technical issues related to the maintenance of civil structures of the organization. Therefore, it is strongly recommended to apply concepts related to "PEOPLE ANALYTICS" to face a varied public, always framed in a strategic context that guarantees the continuity of the project.

It is intended to be followed by other frontline instruments, that is, the most important ones. In the same way, to implement these solutions in other areas of action. It is also expected to evolve the solution currently proposed so that it can make the digital inclusion, for new technologies, of the users of the organization.

\section{REFERENCES}

Bustelo Ruesta, C., and Amarilla Iglesias, R., 2001. Gestión del conocimiento y gestión de la info. Revista PH, 226.

Espinoza, H E., 2006. Gestión de la información engranaje estratégico para la gestión del conocimiento.. "intempres". vol. 15, n. 2006. http://www.bnv.bib.ve/pdf/GestionHeisbellEspinoza.pdf

Jennex, M. E. et al, 2014. Knowledge Management Success in Practice. 47th Hawaii International Conference on System Science, pp. 3615-3624.

Jennex, M.E., 2005. Case Studies in Knowledge Management San Diego State University., USA., Idea Group Publishing.

Marsal, M.; Molina, J. L., 2002. La gestión del conocimiento en las organizaciones", Coleccion de Negocios, Empresa y Economia. Books on the net.

Marulanda Echeverry, C. E. et al, 2012. Modelos de gestión del conocimiento [Knowledge Management Models]. Ventana Informatica, 26.

Murawski, L., 2020. Gamification in human resource management. Status quo and quo vadis. German Journal of Human Resource Management. Zeitschrift für Personalforschung. I-19.

Nonaka, I.; Takeuchi, H., 1995. The knowledge-creating company: how Japanese companies create the dynamics of innovation. New York, Oxford University Press.

Papoutsoglou M., et. al, 2017. Mining People Analytics from StackOverflow Job Advertisements. 43rd Euromicro Conference on Software Engineering and Advanced Applications (SEAA), Vienna, pp. 108-115.

Pérez, S. et al, 2004. Managing knowledge: the link between culture and organizational learning. Journal of Knowledge Management. Vol. 8, núm 6, pp. 93-104.

Rodríguez-Gómez, D., 2006. Modelos para la creación y gestión del conocimiento: una aproximación teórica. Educar, ISSN 0211-819X, № 37, 2006 (Ejemplar dedicado a: La gestión del conocimiento a través de la red), pp. 25-39.

Ruesta, B. C. R. Iglesias, A., 2001. Gestión del Conocimiento y Gestión de la Información, INFORAREA S.L., Boletín del Instituto Andaluz de Patrimonio Histórico, ISSN 1136-1867, Año no 9, $\mathrm{N}^{\circ} 34$ (Ejemplar dedicado a: Especial Monográfico: Museos), pp. 226-230.

Sallis, E.; Jones, G., 2013. Knowledge Management in Education: enhancing leaning and education. Londres: Kogan.

Saz, M., 2001. Gestión del conocimiento: Pros y contras. El profesional de la información, ISSN 1386-6710, Vol. 10, No 4, 2001, pp. 14-28. 10. 10.1076/epri.10.4.14.6527. 